\title{
Combined giant inverse and normal magnetocaloric effect for room-temperature magnetic cooling
}

\author{
Xixiang Zhang, ${ }_{1}^{1} *$ Bei Zhang, ${ }^{1}$ Shuyun Yu, ${ }^{2}$ Zhuhong Liu, ${ }^{2}$ Wenjin Xu, ${ }^{1}$ Guodong Liu, ${ }^{2}$ Jinglan Chen, ${ }^{2}$ Zexian Cao, ${ }^{2}$ and \\ Guangheng $\mathrm{Wu}^{2}$ \\ ${ }^{1}$ Department of Physics, The Hong Kong University of Science and Technology, Clear Water Bay, Kowloon, Hong Kong, \\ People's Republic of China \\ ${ }^{2}$ Beijing National Laboratory for Condensed Matter Physics, Institute of Physics, Chinese Academy of Sciences, \\ Beijing 100080, China
}

(Received 7 June 2007; revised manuscript received 7 September 2007; published 4 October 2007)

\begin{abstract}
In this Brief Report, we report on the observation of a giant positive (inverse) magnetic entropy change (about $28.6 \mathrm{~J} \mathrm{~K}^{-1} \mathrm{~kg}^{-1}$ at $5 \mathrm{~T}$ magnetic field) below $300 \mathrm{~K}$ and a modest negative (normal) entropy change (about $6.6 \mathrm{~J} \mathrm{~K}^{-1} \mathrm{~kg}^{-1}$ at $5 \mathrm{~T}$ ) above $300 \mathrm{~K}$ in a $\mathrm{Ni}_{50} \mathrm{Mn}_{33.13} \mathrm{In}_{13.90}$ single crystal. This temperature-dependent entropy change may suggest that both the magnetizing and demagnetizing processes can be employed for cooling.
\end{abstract}

DOI: 10.1103/PhysRevB.76.132403

PACS number(s): 75.30.Sg, 75.20.En, 75.40.Cx, 75.50.Cc

\section{INTRODUCTION}

In the last two decades, magnetic refrigeration has been demonstrated as a very promising alternative technology, near room temperature, to conventional gas-compression refrigerators because of its unique advantages, such as being environmentally friendly and highly efficient. ${ }^{1-3}$ However, this technology has not been commercialized yet because inexpensive and large magnetocaloric effect (MCE) materials have not been developed, although some giant MCE materials have been discovered in the last decade. ${ }^{4-14}$

It is well known that changes in magnetic entropy are largest near the Curie temperature for a simple ferromagnet due to the fact that ordering of the magnetic spins induced by a magnetic field is the most efficient near the Curie temperature. It has also been accepted that the field-induced, firstorder phase transition in magnetic materials, such as the antiferromagnetic to ferromagnetic transition in FeRh alloys ${ }^{15}$ and paramagnetic to ferromagnetic transitions, ${ }^{4}$ can also give rise to a giant magnetic entropy change. Typically, the giant MCE observed in different systems is closely connected to the field-induced, first-order phase transition. For example, in $\mathrm{LaFeSi}$ alloys, the shift of the paramagnetic to ferromagnetic phase transition to higher temperature in magnetic fields leads to a giant MCE. ${ }^{2,10-12}$ Due to the field-induced, first-order phase transition, giant MCE materials generally exhibit metamagnetism and significant hysteresis loops, which may lead to energy loss and consequently to refrigerant capacity (RC) reductions. ${ }^{7}$ In giant MCE materials, when the applied magnetic field is increased, the magnetic entropy is lowered, i.e., there is a negative entropy change, $\Delta S_{m}<0$. Very recently, a giant "positive" or "inverse" $\Delta S_{m}$ about $18 \mathrm{~J} \mathrm{~K}^{-1} \mathrm{~kg}^{-1}$ for a field change of $5 \mathrm{~T}$ at $300 \mathrm{~K}$ was observed in a $\mathrm{Ni}_{50} \mathrm{Mn}_{37} \mathrm{Sn}_{13}$ compound, a ferromagnetic shape memory alloy (FSMA). This entropy change was ascribed to a first-order martensitic transformation. ${ }^{16}$ Above $300 \mathrm{~K}$, the sign of $\Delta S_{m}$ changes and its magnitude reduces greatly $\left(<4 \mathrm{~J} \mathrm{~K}^{-1} \mathrm{~kg}^{-1}\right)$. The positive $\Delta S_{m}$ indicates that there is a temperature decrease upon application of an external field. Later, the inverse $\Delta S_{m}$ was reported by another group in
MniMnSn (Ref. 17) and NiMnIn (Ref. 18) compounds. Actually, inverse $\Delta S_{m}$ should be a commonly expected phenomenon, when a field-induced phase transition shifts the transition from a high-temperature, high-magnetization phase to a low-temperature, low-magnetization phase to lower temperature. This has been observed previously in other materials, ${ }^{19-22}$ but the size of the inverse $\Delta S_{m}$ is quite small.

$\mathrm{Ni}_{50} \mathrm{Mn}_{50-x} M_{x}(M=\mathrm{In}, \mathrm{Sn}, \mathrm{Sb})$ alloys are FSMA with sharp martensitic transformations and their transformation temperatures strongly depend on the alloy's composition, i.e., the value of $x .^{23}$ The magnetocaloric effect in these alloys, particularly alloys with martensitic transformation temperatures very near the Curie temperatures, which are expected to display a very large entropy change, is very interesting. In this Brief Report, we report on the observation of a giant positive (inverse) magnetic entropy change of about $28.6 \mathrm{~J} \mathrm{~K}^{-1} \mathrm{~kg}^{-1}$ below $315 \mathrm{~K}$, followed by a modest, negative (normal) entropy change of about $6.6 \mathrm{~J} \mathrm{~K}^{-1} \mathrm{~kg}^{-1}$ above $315 \mathrm{~K}$ with an external field increase from 0 to $5 \mathrm{~T}$ $(\Delta H=5 \mathrm{~T})$ in a $\mathrm{Ni}_{50} \mathrm{Mn}_{33.13} \mathrm{In}_{13.90}$ single crystal. This characteristic of the temperature dependence of the entropy changes may suggest that both the magnetizing and demagnetizing processes can be employed for cooling.

\section{EXPERIMENT}

In order to obtain a material with its martensitic transformation temperature near room temperature, the composition of the material was modified from the stoichiometric Heusler alloy. High quality single crystals of $\mathrm{Ni}_{50} \mathrm{Mn}_{50-x} \mathrm{In}_{x}$ were grown at a rate of $5-30 \mathrm{~mm} / \mathrm{h}$ using a Czochralski instrument with a cold crucible system. The starting material was prepared from Ni, Mn, and In with purity of $99.95 \%$. The structure of single crystal was determined by x-ray diffraction performed on a Rigaku D/Max 2400 using the $\mathrm{Cu} K \alpha$ irradiation at room temperature and $93 \mathrm{~K}$. It reveals that the high-temperature phase is L21-type ordered structure for this off-stoichiometric compound with the lattice parameter of $6.006 \AA$. Cooling to $93 \mathrm{~K}$, the crystal structure changes to orthorhombic structure with a rather complex 


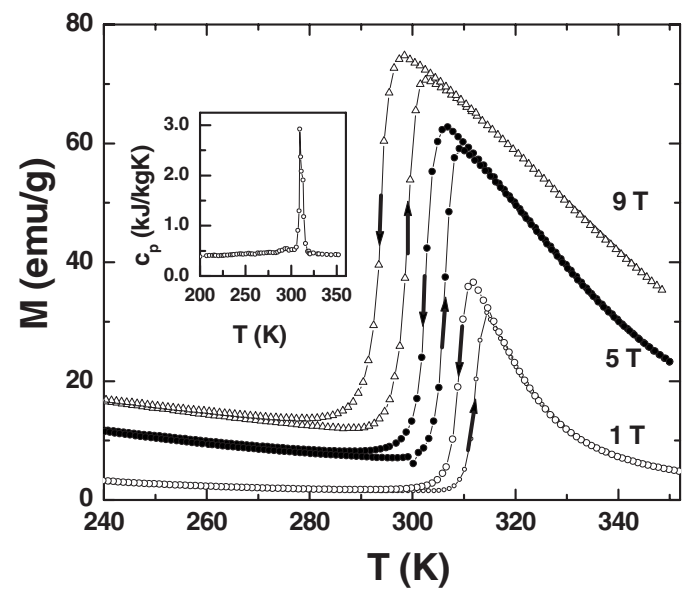

FIG. 1. Temperature-dependent magnetization measured in the cooling and warming processes with magnetic fields of 1,5 , and $9 \mathrm{~T}$. The inset is the zero-field specific heat of the crystal.

martensitic modulated substructure. The magnetic properties of these crystals were measured using a Quantum Design superconducting quantum interference device magnetometer (5-390 K, with maximum field of $5 \mathrm{~T}$ ) and a Quantum Design physical property measurement system (PPMS) $(10-330 \mathrm{~K}$, with maximum field of $9 \mathrm{~T})$. The heat capacity of the crystal was measured using the same Quantum Design PPMS.

\section{RESULTS AND DISCUSSIONS}

All of the crystals exhibit sharp martensitic transformations from ferromagnetic austenite to ferrimagnetic or paramagnetic martensite, and their transformation temperatures depended critically on the composition. ${ }^{22}$ Magnetic and electrical measurements revealed that all of them had a Curie temperature of about $315 \mathrm{~K}$, although their martensitic transformation temperature increased from 150 to $350 \mathrm{~K}$ as $x$ changes from 16.3 to $14{ }^{22,24}$ The field-induced phase transition that shifts the martensitic transformation to a lower temperature was observed in all the crystals. ${ }^{22}$ Due to the fieldinduced phase transition, positive entropy changes $\left(\Delta S_{m}\right.$ $>0)$ are observed. The NiMnIn crystal with $x=14.7$ showed a martensitic transition at $310 \mathrm{~K}$, very near its Curie temperature. We report the results obtained from this crystal.

Figure 1 shows the temperature-dependent magnetization curves measured during the cooling and warming processes with different magnetic fields, $H=1,5$, and $9 \mathrm{~T}$. It is clear that the magnetic nature of the crystal varies with temperature. The abrupt changes in magnetization at around $300 \mathrm{~K}$ with varying temperature show a clear thermal hysteretic behavior, strongly indicating a martensitic transition. The much lower magnetization of the martensite suggests a very weak magnetic phase. ${ }^{25}$ The decrease of magnetization with temperature above the peaks can also be fitted by the CuriesWeiss law, $M(T)=C /\left(T-T_{0}\right)$, perfectly with $T_{0}=315.04 \mathrm{~K}$. The Curie temperature of ferromagnetic austenite is about $315 \mathrm{~K}$, which was also deduced from both the $H / M$ vs $M^{2}$ plots of the isothermals. To confirm the martensitic transfor-

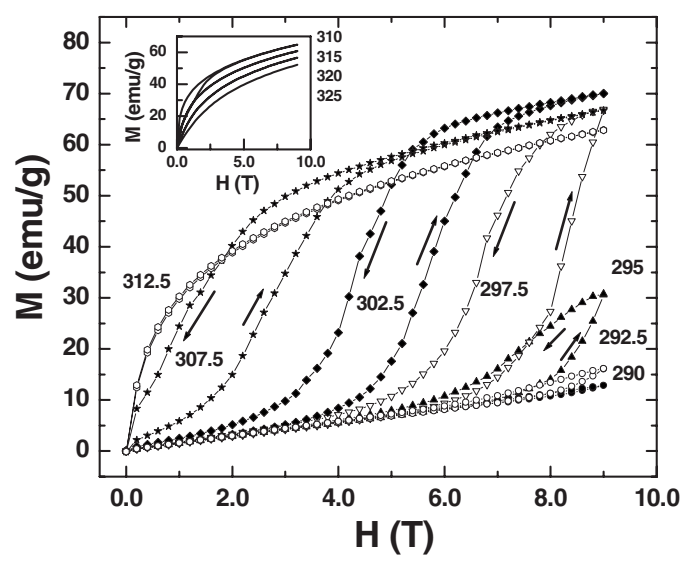

FIG. 2. Representative isothermal magnetization curves measured near the martensitic transition temperature.

mation, we carried out a heat capacity measurement from 3 to $350 \mathrm{~K}$ as the temperature increased in a zero magnetic field, as shown in the inset of Fig. 1. We observed a narrow, sharp peak in the heat capacity at $310 \mathrm{~K}$, which indicates a first-order phase transition. The sharp peak is in agreement with the results reported by Sutou et al. ${ }^{23}$ that the transformation from the martensite to austenite phase is an endothermic process.

The most intriguing feature in Fig. 1 is that the martensitic transformation (the weak-magnetic to ferromagnetic transformation) can be shifted by magnetic field, e.g., it was shifted downward about $16 \mathrm{~K}$ with the $9 \mathrm{~T}$ magnetic field. A large entropy change should therefore be expected. The relatively large shift of the transformation temperature is due to the large magnetization difference, $\Delta M$, between the two phases, e.g., the $\Delta M$ is larger than $60 \mathrm{emu} / \mathrm{g}$ (at $9 \mathrm{~T}$ ). This transformation is strikingly different from what is observed in other giant MCE materials such as GdGeSi alloys ${ }^{4,5}$ and other giant MCE alloys, ${ }^{8-15}$ where the field-induced phase transition shifts the transformation temperature to a high temperature and gives rise to a giant negative magnetic entropy change. Here, we observe a positive magnetic entropy change in this material as also observed in this family. ${ }^{16,18}$

To obtain the entropy change, we have measured the isothermal magnetization in the temperature range of 5-390 K. Shown in Fig. 2 are the representative isothermals near the martensitic transition. The interesting feature in Fig. 2 is the sharp changes in the magnetization at the different critical fields in both the magnetizing and demagnetizing processes. These critical fields strongly depend on the temperature at which the isothermals were measured. These features strongly suggest the metamagnetic nature of the material. The magnetic entropy change was then calculated from the isothermal data. Based on the Maxwell relations, the magnetic entropy change for the isobaric-isothermal system is calculated by

$$
\Delta S_{m}=\int_{0}^{H}\left(\frac{\partial M}{\partial T}\right)_{H} d H
$$

When the magnetization is measured at discrete fields and temperature intervals, the magnetic entropy change can be 

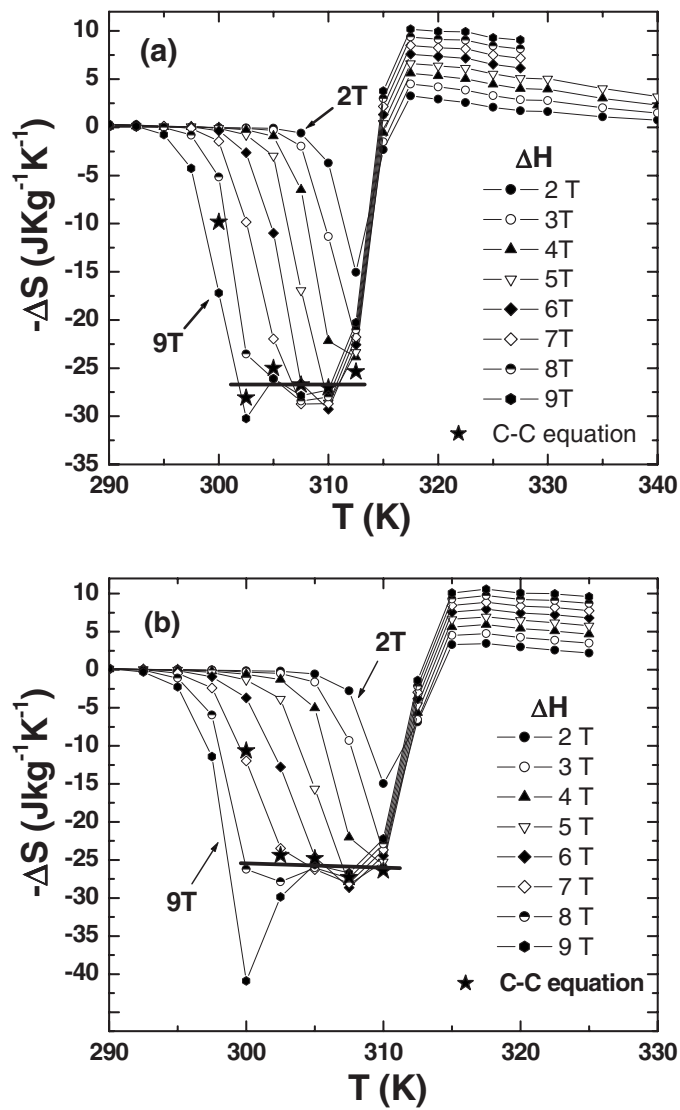

FIG. 3. The entropy change as a function of the temperature under different magnetic field variations $(\Delta H)$. Calculated entropy change using (a) the magnetizing curves and (b) the demagnetizing curves. Since the crystal is in the paramagnetic state, the magnetizing and demagnetizing curves are identical, and some isothermals were measured only up to $5 \mathrm{~T}$. The stars are the calculated entropy changes using the Clausius-Clapeyron equation.

calculated approximately using the following numerical formula: ${ }^{26}$

$$
-\Delta S_{M}=\sum_{i} \frac{1}{T_{i+1}-T_{i}}\left(M_{i}-M_{i+1}\right) \Delta H_{i}
$$

where $M_{i}$ and $M_{i+1}$ are the magnetization values obtained at temperatures $T_{i}$ and $T_{i+1}$ in a field $H$, respectively. As discussed by Provenzano et al. ${ }^{7}$ the error in the calculated $\Delta S_{m}$ is about $1 \%$ or less for a field variation $\Delta H=5 \mathrm{~T}$. For $\Delta H$ $=9 \mathrm{~T}$, the error in $\Delta S_{m}$ should not be much larger than $1 \%$. Both the magnetizing and demagnetizing isothermals were used to calculate $\Delta S_{m}{ }^{5}$

Shown in Fig. 3 are data of $\Delta S_{m}$ for different magnetic field variations $(\Delta H)$ in the temperature range of 290-340 K. The most striking feature of the entropy change is the giant, positive or inverse entropy change in the range of $295-315 \mathrm{~K}$ with magnitudes of 28.6 and $28.2 \mathrm{~J} \mathrm{~K}^{-1} \mathrm{~kg}^{-1}$ for the respective magnetizing and demagnetizing processes at $5 \mathrm{~T}$, which are much larger than $18 \mathrm{~J} \mathrm{~K}^{-1} \mathrm{~kg}^{-1}$ observed in NiMnSn alloys. ${ }^{16}$ This value is similar to that observed in NiCoMnIn alloys. ${ }^{25}$ The spike values for $\Delta H=9 \mathrm{~T}$ are 30.2 and $40.9 \mathrm{~J} \mathrm{~K}^{-1} \mathrm{~kg}^{-1}$ for magnetizing and demagnetizing processes, respectively, which are much larger than $13 \mathrm{~J} \mathrm{~K}^{-1} \mathrm{~kg}^{-1}$ observed in a similar alloy with $\mathrm{Ni}_{46} \mathrm{Mn}_{41} \mathrm{In}_{13}$ composition. ${ }^{27}$ This giant, positive $\Delta S_{m}$ must be associated with the field-induced weak-magnetic to ferromagnetic phase transition, which shifts the transition to lower temperatures. This field-induced magnetic phase transformation should also be accompanied by a magnetocrystallographic transformation as observed recently in similar alloys. ${ }^{25}$ This crystallographic transformation changes the crystal lattice or the arrangement of the atoms in the crystal, consequently altering the exchange coupling of the magnetic atoms, leading to ferromagnetic coupling in the austenite phase. Since the giant, positive $\Delta S_{m}$ is a result of the field-induced first-order phase transition, its values have also been calculated by using the Clausius-Clapeyron equation, ${ }^{5,25}$

$$
\left|\frac{\Delta \theta}{\Delta H}\right|=\left|\frac{\Delta M}{\Delta S}\right|=\text { const, }
$$

where $\theta$ is the transformation temperature at field $H$ and $\Delta M$ and $\Delta S$ are the difference between the magnetizations and the difference between the entropies of the two phases, respectively. The calculated values of $\Delta S_{m}$ at $9 \mathrm{~T}$ were also plotted in Fig. 3 as the stars, which is in reasonably good agreement with that calculated using the Maxwell equation.

Another very important feature in Fig. 3 is that at $315 \mathrm{~K}$, $\Delta S_{m}$ changes its sign and drops to about 6.6 and $10 \mathrm{~J} \mathrm{~K}^{-1} \mathrm{~kg}^{-1}$ for $\Delta H=5$ and $9 \mathrm{~T}$, respectively. The negative $\Delta S_{m}$ is due to the fact that the alloy has a Curie temperature of $315 \mathrm{~K}$, above which its magnetization decreases monotonically with increasing temperature. The RC values were calculated by numerically integrating the area under the $\Delta S_{m}(T)$ curve (Fig. 3) and taking the temperatures at which $\Delta S_{m}(T)$ is half of the peak of $\Delta S_{m}$ as the integration limits. ${ }^{7,26,28}$ For the positive entropy change of $\Delta H=9 \mathrm{~T}$, the $\mathrm{RC}$ values are 341 and $342 \mathrm{~J} \mathrm{~kg}^{-1}$ for magnetizing and demagnetizing processes, respectively. For the negative $\Delta S_{m}$, the RC value is about $125 \mathrm{~J} \mathrm{~kg}^{-1}$ for $\Delta H=5 \mathrm{~T}$.

The most important characteristic of the $\Delta S_{m}(T)$ curve shown in Fig. 3 is that the giant positive entropy change is followed by a significantly large negative entropy change with increasing temperature, which may strongly suggest that both the magnetizing and demagnetizing processes can be employed for cooling. As it is well known, a conventional ferromagnet or a conventional giant MCE material, in which the external field shifts the phase transition to a higher temperature, can only give rise to a negative or "normal" $\Delta S_{m}(T)$, and that only the demagnetization process can be utilized for cooling, if these materials are used as magnetic refrigerants. If both the magnetizing and demagnetizing processes can be utilized for cooling, the efficiency may be greatly increased because both processes consume energy.

The magnetic refrigerator based on the characteristic of the entropy change is schematically shown in Fig. 4. The working principle (or process) is briefly described as the following. (1) Apply a magnetic field $H_{S}(=5 \mathrm{~T})$ to the chamber that contains NiMnIn beads, cooling medium, and a heat exchanger that is connected to a thermal reservoir at tem- 


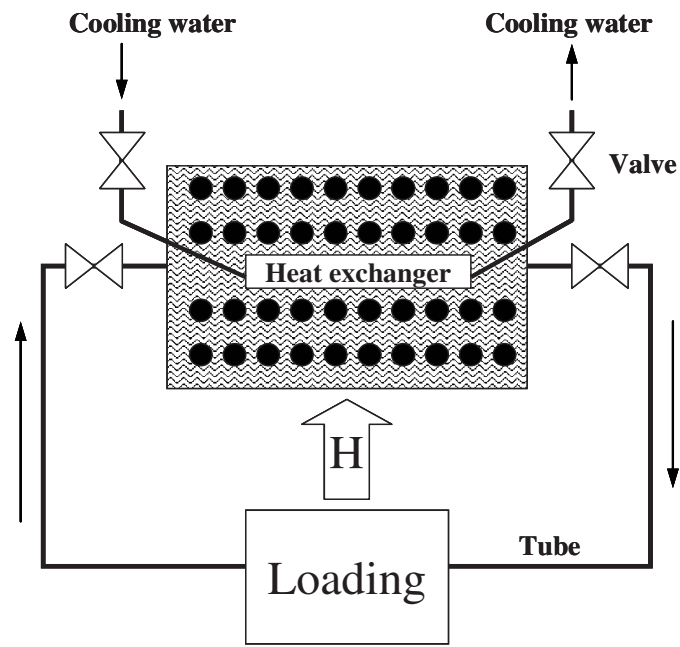

FIG. 4. The schematic drawing of the proposed magnetic refrigerator.

perature $T_{S} \sim 315 \mathrm{~K}$ (isothermal magnetizing process). (2) Pump out the liquid from the heat exchanger and turn off magnetic field, which lowers the temperature in the chamber (NiMnIn beads and cooling medium) to $T_{1}(<315 \mathrm{~K})$ due to the normal entropy change (adiabatic demagnetizing process). (3) Turn on the magnetic field $H_{S}$ again; the chamber will be further cooled to $T_{2}$ due to the positive entropy change in this temperature range (adiabatic magnetizing pro- cess or magnetic-field-induced phase transition). (4) Pump the cooling medium into the loading at $T_{3}\left(>T_{2}\right)$. (5) After the liquid absorb heat in the loading, pump the cooling medium back to the chamber. (6) Flow the liquid in thermal reservoir through the heat exchanger in the chamber to stabilize the temperature in the chamber to $T_{S}$, (the materials transform into paramagnetic phase and the heat was brought away by the heat exchanger). (7) Turn off the magnetic field $H_{S}$ and repeat steps (1)-(6). It is clear that in one cycle, both magnetizing and demagnetizing processes are utilized for cooling, which will certainly increase the efficiency of the magnetic refrigerator and save energy. By tuning the composition, the martensitic transformation temperature can be adjusted to fit the requirement of the applications.

In summary, we have observed a giant, positive magnetic entropy change followed by a significantly large negative change with increasing temperature. This unique feature is due to that fact that a field-induced phase transition from the paramagnetic martensite phase to the ferromagnetic austenite phase is very near the Curie temperature of the ferromagnetic austenite phase.

\section{ACKNOWLEDGMENTS}

This work was supported by grants from the Research Grants Council of the Hong Kong Special Administration Region, China. The authors would like to thank T. S. Zhao for the useful discussions. *phxxz@ust.hk

${ }^{1}$ J. Glanz, Science 279, 2045 (1998).

${ }^{2}$ K. A. Gschneidner, V. K. Pecharsky, and A. O. Tsokol, Rep. Prog. Phys. 68, 1479 (2005).

${ }^{3}$ A. M. Tishin and Y. I. Spichkin, The Magnetocaloric Effect and its applications (IOP, Bristol, 2003).

${ }^{4}$ V. K. Pecharsky and K. A. Gschneidner, Phys. Rev. Lett. 78, 4494 (1997).

${ }^{5}$ A. Giguere, M. Foldeaki, B. R. Gopal, R. Chahine, T. K. Bose, A. Frydman, and J. A. Barclay, Phys. Rev. Lett. 83, 2262 (1999).

${ }^{6}$ O. Tegus, E. Bruck, K. H. J. Buschow, and F. R. de Boer, Nature (London) 415, 150 (2002).

${ }^{7}$ V. Provenzano, A. J. Shapiro, and R. D. Shull, Nature (London) 429, 853 (2004).

${ }^{8}$ X. X. Zhang, J. Tejada, Y. Xin, G. F. Sun, K. W. Wong, and X. Bohigas, Appl. Phys. Lett. 69, 3596 (1996).

${ }^{9}$ Z. B. Guo, Y. W. Du, J. S. Zhu, H. Huang, W. P. Ding, and D. Feng, Phys. Rev. Lett. 78, 1142 (1997).

${ }^{10}$ X. X. Zhang, G. H. Wen, F. W. Wang, W. H. Wang, C. H. Yu, and G. H. Wu, Appl. Phys. Lett. 77, 3072 (2000).

${ }^{11}$ F. X. Hu, B. G. Shen, J. R. Sun, Z. H. Cheng, G. H. Rao, and X. X. Zhang, Appl. Phys. Lett. 78, 3675 (2001).

${ }^{12}$ A. Fujita, S. Fujieda, Y. Hasegawa, and K. Fukamichi, Phys. Rev. B 67, 104416 (2003).

${ }^{13}$ S. Gama, A. A. Coelho, A. de Campos, A. M. G. Carvalho, F. C. G. Gandra, P. J. von Ranke, and N. A. de Oliveira, Phys. Rev. Lett. 93, 237202 (2004).

${ }^{14}$ D. H. Ryan, M. Elouneg-Jamroz, J. van Lierop, Z. Altounian, and H. B. Wang, Phys. Rev. Lett. 90, 117202 (2003).
${ }^{15}$ M. P. Annaorazov, S. A. Nikitin, A. L. Tyurin, K. A. Asatryan, and A. K. Dovletov, J. Appl. Phys. 79, 1689 (1996).

${ }^{16}$ T. Krenke, E. Duman, M. Acet, E. F. Wassermann, X. Moya, L. Manosa, and A. Planes, Nat. Mater. 4, 450 (2005).

${ }^{17}$ Z. D. Han, D. H. Wang, C. L. Zhang, H. C. Xuan, B. X. Gu, and Y. W. Du, Appl. Phys. Lett. 90, 119902 (2007).

${ }^{18}$ Z. D. Han, D. H. Wang, C. L. Zhang, S. L. Tang, B. X. Gu, and Y. W. Du, Appl. Phys. Lett. 89, 122503 (2006).

${ }^{19}$ P. J. von Ranke, V. K. Pecharsky, K. A. Gschneidner, and B. J. Korte, Phys. Rev. B 58, 14436 (1998).

${ }^{20}$ H. Wada, Y. Tanabe, K. Hagiwara, and M. Shiga, J. Magn. Magn. Mater. 218, 203 (2000).

${ }^{21}$ P. Chen, Y. W. Du, and G. Ni, Europhys. Lett. 52, 589 (2000).

${ }^{22}$ S. Y. Yu, Z. H. Liu, G. D. Liu, J. L. Chen, Z. X. Cao, G. H. Wu, B. Zhang, and X. X. Zhang, Appl. Phys. Lett. 89, 162503 (2006).

${ }^{23}$ Y. Sutou, Y. Imano, N. Koeda, T. Omori, R. Kainuma, K. Ishida, and K. Oikawa, Appl. Phys. Lett. 85, 4358 (2004).

${ }^{24}$ B. Zhang, X. X. Zhang, S. Y. Yu, J. L. Chen, Z. X. Cao, and G. H. Wu, Appl. Phys. Lett. 91, 012510 (2007).

${ }^{25}$ R. Kainuma et al., Nature (London) 439, 957 (2006).

${ }^{26}$ M. Foldeaki, R. Chahine, and T. K. Bose, J. Appl. Phys. 77, 3528 (1995).

${ }^{27}$ K. Oikawa, W. Ito, Y. Imano, Y. Sutou, R. Kainuma, K. Ishida, S. Okamoto, O. Kitakami, and T. Kanomata, Appl. Phys. Lett. 88, 122507 (2006).

${ }^{28}$ K. A. Gschneidner, V. K. Pecharsky, A. O. Pecharsky, and C. B. Zimm, Mater. Sci. Forum 315-3, 69 (1999). 